\title{
Defining Product Lifecycle Management: Comparing the Perspectives of Users and Consultants
}

\author{
William Neil Littell* \\ Engineering Technology and Management, Russ College of Engineering at Ohio University, Athens, USA \\ *Corresponding author: littellw@ohio.edu
}

\begin{abstract}
Product Lifecycle Management (PLM) is a strategy that is being leveraged by many companies to improve both the execution of new product development as well as the general operations of the company. Because the breadth of PLM is so wide, many companies struggle with defining PLM. This lack of a definition causes confusion within the company that is confounded by the many definitions supplied by consultants and solution providers. This paper seeks to develop a user centric definition of PLM, and offers guidance for individuals implementing a PLM strategy.
\end{abstract}

Keywords: Product Lifecycle Management, PLM, PDM, ERP, CRM, SCM

Cite This Article: William Neil Littell, "Defining Product Lifecycle Management: Comparing the Perspectives of Users and Consultants." Journal of Business and Management Sciences, vol. 6, no. 3 (2018): 76-81. doi: 10.12691/jbms-6-3-2.

\section{Introduction}

Manufacturing companies typically strive to produce products with advanced features more rapidly than their competitors. As a result of the pressures of competition, many companies have turned to Product Lifecycle Management (PLM) as a general strategy for improving both new product development (NPD) and operations management [1]. Research has shown that up to $70 \%$ of a products cost is determined by activities that take place within the NPD process within a company [2]. This fact helps give insight into why companies are rapidly investing in PLM technologies. In 2016, the PLM industry grew to 40.6 Billion USD, with a compound annual growth rate (CAGR) projected to be at $6.7 \%$ according to CimData [3]. Companies implementing PLM solutions are finding much value in terms of speed to market, reduction of errors, and reducing both development and manufacturing costs. With many companies, consultants and software providers working in the PLM space, many have developed their own ad hoc definition for PLM. The lack of a common, user centric, definition creates confusion in the marketplace. Despite the fact that PLM has received much attention over the past twenty years, there exists a void with respect to the actual definition of PLM [4].

\section{History and Context of PLM}

Computational solutions provide methods to automate and streamline data management, delivery, and reporting. This automation enables a competitive advantage to many companies who wish their employees to be able to focus on solving problems and generating new ideas. Software companies have focused on providing pointed enterprise systems, with a focus on specific areas of the business enterprise. Enterprise Resource Planning (ERP) encompasses typical transactional management of discrete data, such as the comprehensive management of parts inventories. Supply Chain Management (SCM) integrates suppliers into the business enterprise, Customer Resource Management (CRM) enables the company to manage the marketing and service relationship between the consumer and the company [5]. Product Lifecycle Management (PLM) for many companies is the newest of the four enterprise systems acronyms. PLM for many companies describes the tools and techniques used to manage data across the extended enterprise with respect to product data. However, this definition is purposefully vague, as the focus of this article is discovering a user centric definition of PLM.

The requirement for companies to implement a PLM solution derives from the desire to integrate all corporate knowledge into one master data management strategy. While each of the systems identified above contributes to the MDM strategy, the strategy is incomplete without the actual product data. Because the other systems (ERP, SCM, and CRM) leverage and transact upon data that is centric to the product, it makes sense to include the systems that actually controls the product definition in the corporate vision of the MDM strategy [6].

PLM methodologies have grown as an extension of product data management systems (PDM), which were originally developed to manage data, specifically computer aided design data. Modern manufacturing companies employ teams of people who each contribute to the design of a product $[7,8]$. Collaboration challenges related to the general management of the many files created by people yielded the need for a centralized repository to house the engineering data, as well as revision control the data. This yielded the first PDM 
systems, which operated as a database connected to a vault. These systems allow team members to effectively collaborate through automatic revision control of data. PDM systems quickly were extended to include technologies such as automated workflows, which allow individuals to route approvals of documents through others within the company. This technology replaced manual and paper based processes within these companies, which yielded many improvements related to processing product data as required by different departments related to the new product development process [9]. However, PDM implementations were generally limited to teams of people who were collocated.

CAD companies quickly embraced PDM systems as these systems form a synergy which greatly improves the organization and flow of work within companies which lack such a system. These synergies were leveraged by the CAD companies to sell both CAD and PDM systems as one package to rapidly add value to a company. As technology continued to advance within both CAD and PDM, the systems began to incorporate other technologies related to the product definition. As new technologies began to merge into the PDM ecosystem, PDM became a term which did not accurately describe what was being actively managed [10]. In 2000, on the Dassault Systemes coined the term Product Lifecycle Management to more accurately describe the shift of technology from managing product data to managing the product lifecycle [11]. Companies who embrace PLM are able to leverage engineering data for purposes such as the development of work instructions and service documentation. Analysis and simulation data is tied to the product data, and manufacturing programs are tied to individual parts. As myriad data was added to the PDM system, companies realized that the management of data was a component of managing the lifecycle of the products they were producing. Product Lifecycle Management (PLM) became the term used to describe the active management of product data across the extended enterprise [10]. Today, state-of-the-art PLM systems can manage a digital twin of the products and the facilities where they are produced. Engineers can impose simulated physical forces, simulate the passage of time, as well as morph the digital twin of products to generate derivative designs and simulate their production virtually before the design is implemented in the real world.

The promise of PLM has been increased utilization of engineering data for applications within other departments, with the intent to improve overall operations across the enterprise. This means that there likely is not one solution that fits all companies perfectly. Companies will likely have one PLM system, which is required to plug into other systems to ensure the best fit for the company [12]. Companies leverage intelligence built into the context of the design of a product to allow systems to proactively respond to design changes. For example, if a user updates the geometry of a part, the code used to drive the machines to produce the parts can automatically be updated to reflect the change. Supplementary documentation such as work instructions and service literature can be systematically updated to reflect these changes as well. Through building an intelligent PLM architecture, errors are reduced through the automated implementation of part and manufacturing rules. By actively driving processes within the company from the engineering data, the company can more easily react to new product development as well as engineering changes on existing products. Because the data is managed from one system, there exist a single source of truth for the company. Communication of changes becomes actively pushed by the PLM system instead of passively pulled by the departments seeking to catch up or react to changes after they have been implemented. This automated coordination of information typically results in increased data processing time as well as reduced scrap and rework [6]. Cantamessa, Montagna, \& Neirotti, [13] discovered through their study of PLM implementations within Aerospace companies that the main benefit of the implementation was the freedom provided to employees with respect to being able to find information.

\section{Solution Provider Definitions}

There are many companies who claim to sell PLM solutions. However, when one reviews the state of the industry and what is being sold in the PLM realm, four companies dominate. These companies include Dassault Systèmes, PTC, Siemens, and AutoDesk [8,14]. It should be no surprise considering the legacy of PLM growing from CAD to PDM to PLM that each of these four PLM companies that also sell computer aided design (CAD) solutions as a component of their comprehensive PLM suite of tools. These companies are focused on providing comprehensive PLM solutions to companies, not discrete software which plugs into an existing PLM implementation.

\subsection{Dassault Systèmes}

Even though Dassault Systèmes was the first company to use the term Product Lifecycle Management [6], a definition of PLM remains elusive on the company website. To gain some perspective of the corporate Dassault definition, the author was able to find an interview with the past Director of Dassault Americas, who stated:

Q. Product lifecycle management (PLM) is the core product at Dassault Systems. How would you define PLM?

A. The most important word of those three is lifecycle. That's what makes it more than just design. It's managing the lifecycle of an innovation, from when it starts with a concept, an idea, and goes into requirements, definition, and the design phase. Then you have to simulate it. You can even simulate the manufacturing environment. After that we can track changes, parts changes, and defects.[15]

\subsection{Siemens PLM}

Siemens PLM is headquartered in Plano Texas, and is a part of the Siemens Industry Automation Division. Their three-dimensional CAD package is branded as Siemens NX, and their PLM package is known at Teamcenter. These products are advertised to provide a comprehensive engineering design, simulation, and collaborative solution [16]. Siemens is explicit with their definition of PLM as stated on their corporate website: 
Product lifecycle management (PLM) is an information management system that can integrate data, processes, business systems and, ultimately, people in an extended enterprise. PLM software allows you to manage this information throughout the entire lifecycle of a product efficiently and cost-effectively from ideation, design and manufacture through service and disposal. [17]

\subsection{Parametric Technology Company PTC}

Parametric Technology Company, or PTC offers a parametric CAD package called Creo, which can be managed by their PLM tool called Windchill. While an explicit definition of PLM from PTC could not be found, the marketing verbiage for their PLM offering provides a glimpse of the PTC vision of PLM:

Windchill offers breakthrough Product Lifecycle Management (PLM) capabilities, unleashing more data to more stakeholders throughout your organization through a single source of truth for product data and processes. [18]

\subsection{AutoDesk}

AutoDesk is the newest of the big four to provide a comprehensive PLM solution to customers. The AutoDesk definition of PLM is explicit as is published on their website:

Product lifecycle management (PLM) is the process of managing complex product information, engineering and manufacturing workflows, and collaboration.

PLM software connects people, processes, and data across the entire product lifecycle to a central repository of information. So everyone from the conceptual designer to the end-customer is on the same page, sharing the same up-to-date product definition. [19]

Of the big four companies, only Siemens and AutoDesk explicitly provide a definition for PLM. Perhaps this is because these companies realize that defining PLM can be difficult, and therefore they allow their business partners and salespeople the freedom to provide a generic PLM vision instead of an explicit definition which may be polarizing to some companies.

Upon analysis of the four definitions provided, one can see some trends within the definitions provided by each of the companies. For example, each company talks about people being able to share data. Each definition also addresses the concept of managing the processes which implement the data into physical product through the life of the product; from conception to disposal. The definitions also (either imply or explicitly) address the requirement of a database, or a single source of the data that all users access to collaborate.

\section{Consultant Definitions of PLM}

Three of the most recognized consultant definitions of PLM include those of Stark and Grieves [9] and CimData.

The CimData definition of PLM is one of the most comprehensive definitions of PLM. Existing of 231 words, the CimData definition emphasizes that PLM is a business approach to resolving some of the difficult issues related to comprehensively managing product data. The CimData definition further defines the control of data across the entire (even suppliers) enterprise across the life of the product. The state that PLM is "Integrating people, processes, business systems, and information". Also included in this definition are three concepts which are fundamental to data management with respect to PLM. These concepts include 1. Managing access to product data, 2. Ensuring integrity of the data, and 3. Actively managing the processes used within the lifecycle of the data (from creation to retirement of the data).

John Stark is perhaps the most published and referenced of the PLM authors. His three books (with a fourth one coming soon) on the topic have collectively been referenced over 1000 times. John also owns a PLM consulting company, which provides services to the big four PLM companies as well as some of the largest and most prestigious PLM implementing companies in the world. John's definition of PLM is:

- Product Lifecycle Management (PLM) is defined as the business activity of managing, in the most effective way, a company's products all the way across their lifecycles; from the very first idea for a product all the way through until it is retired and disposed of.

- PLM is the management system for a company's products. PLM manages the whole product range, from individual part through individual product to the entire portfolio of products.

- At the highest level, the objective of PLM is to increase product revenues, reduce product-related costs, maximise the value of the product portfolio, and maximise the value of current and future products for both customers and shareholders [20].

Following John Stark, Michael Grieves is a foremost authority on PLM. Michael states that "Product Lifecycle Management (PLM) is an integrated, information-driven approach comprised of people, processes/practices, and technology to all aspects of a product's life and its environment, from its design through manufacture, deployment and maintenance - culminating in the product's removal from service and final disposal. (2012 p.229)"

\section{Disagreement between Definitions}

In their paper, Corallo, Latino, Lettera, Marra and Verardi [21] compared definitions of PLM from 17 different sources across the three aspects of PLM mentioned above, but also across several subclassifications within each general PLM theme. These authors formed a PLM community of practice with a mission to develop a holistic definition of PLM in an attempt to clarify the different themes they observed. Of the definitions of PLM analyzed, the definition from CimData was determined to be the most complete, as it addressed a total of 9 out of a total of 10 PLM definition subcategories.

However, it was observed by these authors that there exists a void with respect to the inclusion of the concept of what they call a "unique and timed product data source" as well as a void with respect to explicitly stating PLM solution "consistency, traceability, and long-term 
archiving" [21] p.9. While it is true that these components of PLM definitions were explicitly missing, it can be argued that both of these components are intrinsic to the nature of the definition. For example, many definitions of PLM mention managing or controlling data. It can be assumed that if the data is managed or controlled, it can be produced to the users when needed, and that traceability and long-term archival of the data exists, thereby nullifying the void as previously identified.

\section{Why is Defining PLM an Important Problem?}

Defining PLM is an important issue for many companies, because the definition communicates the goals of the tools, methods, and technologies that are being implemented as well as the desired results of the implementation. However, the many different definitions of PLM being discovered by companies who are exploring PLM as a potential solution to help their specific business is overwhelming. While some of the information seems to be fixated on communicating a message of future looking integration (PTC), others seem to avoid defining PLM altogether (Dassault and PTC) perhaps to allow customers the freedom to implement their vision of PLM. The dissonance between PLM vendors is striking. Consultants defining PLM seem to be wary of focusing on any single product. This makes sense as each of the consultants featured in this article work with many companies, and with practically all of the PLM solutions available on the market. While both the consultants and companies definitions of PLM do share common characteristics, to date, there have not been any surveys to actual users soliciting their personal definition of PLM. The results in this study will resolve this void.

The confusion over the definition of PLM results from the existence of many PLM tools, service providers, and even across different industries implementing a PLM strategy, which yields difficulty in defining a holistic PLM strategy $[4,21]$. PLM technologies are evolving so quickly, that CIOs are having trouble keeping up with the technology. This yields vendors who promote discreet products as a complete PLM package. This practice potentially allows vendors to twist definitions to promote specific software functions, which the vendors then boast about being the market leader to earn sales [12]. The goal of this paper is to investigate the definition of PLM from the perspective of both consultants and users in an attempt to understand the different perspectives of PLM.

There are many companies who claim to sell PLM solutions. However, to delimit this study, the author limited recruitment of subjects to individuals either working for or using PLM companies that also sell computer aided design (CAD) solutions as a component of their PLM suite of tools. This is because these companies products are focused on providing comprehensive PLM solutions to companies, not discrete software which plugs into an existing PLM implementation. These companies include Dassault Systemes, PTC, Siemens, and AutoDesk. This sentiment is echoed by the online PLM blog tech-clarity.com, who refers to these four companies as the big four when it comes to PLM [14].

\section{Investigating Perceptions between Expert Users and Consultants}

The author approached employees from each of these four companies and informed them of the survey. Each company had at least one participant in the survey, but not more than four participants. The author recruited users from each of these companies by posting within the forums of the respective user groups as well as contacting the user groups directly to help identify users. A total of 11 consultants were recruited across the big four PLM companies, PLM researchers, and experts and a total of 9 users were recruited across solution users.

The responses of the participants were combined into response data, and this data was input into wordclouds.com to generate the illustrations in this article. The conversational data was systematically processed to remove nondescript words such as prepositions and conjunctions, leaving behind only the words which communicate intent. From this list of words, the author combined similar words in order to more accurately count the frequency of occurrence of an individual thought. For example, the word product may have appeared in the written response as: product, products, Product or product's. In cases such as these, the total word count was combined into the form of the word that appeared most frequently in the preprocessed data. For example, in the consultants cohort of this study, the word product appeared 12 times. Product $(\mathrm{n}=6)$, product's $(\mathrm{n}=5)$, and products $(\mathrm{n}=5)$ were combined with the first instance of the word product and the total frequency for the word cloud was weighted at 28 instances for product. The author also omitted words that only occurred once or twice during the dialog of the users as these words were determined to be insignificant to a holistic definition.

\subsection{Consultants}

11 consultants provided a total of 462 words when asked to define PLM in their own words. The results of their comments are visualized in the word cloud in Figure 1.

Table 1. Responses for Consultants Defining PLM

\begin{tabular}{|c|c|}
\hline Occurrence & Consultants \\
\hline 28 & Product \\
\hline 18 & PLM \\
\hline 15 & Lifecycle \\
\hline 15 & Management \\
\hline 11 & processes \\
\hline 9 & data \\
\hline 6 & software \\
\hline 6 & system \\
\hline 5 & creating \\
\hline 5 & tools \\
\hline 4 & enterprise \\
\hline 4 & set \\
\hline 4 & support \\
\hline 3 & information \\
\hline 3 & throughout \\
\hline 3 & ideation \\
\hline 3 & business \\
\hline 3 & process \\
\hline 3 & people \\
\hline 3 & using \\
\hline 3 & class \\
\hline 3 & manufacturing \\
\hline 3 & exist \\
\hline
\end{tabular}




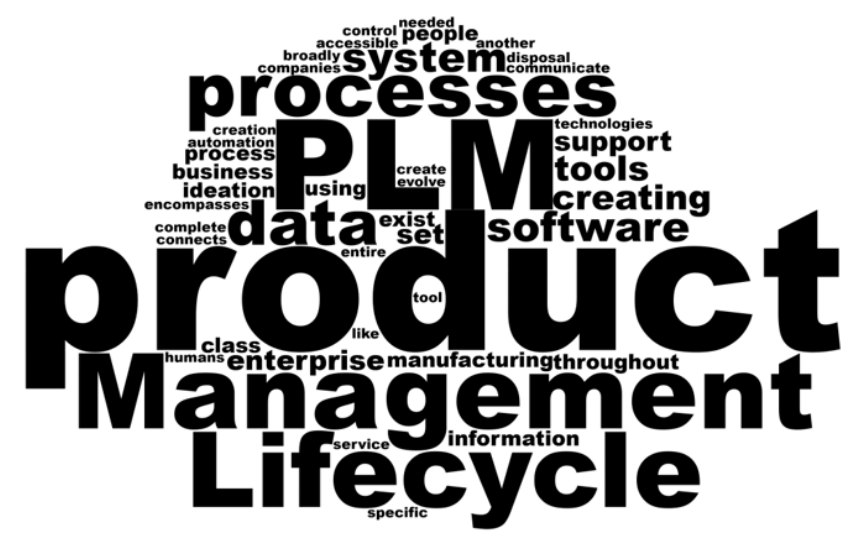

Figure 1. A consolidated word cloud analyzing word frequency for consultants when asked to define PLM

\subsection{Expert Users}

9 Users provided a total of 355 words for analysis.

Table 2: Word Use Responses for Expert Users Defining PLM

\begin{tabular}{|l|l|}
\hline Occurrence & Users \\
\hline 13 & Product \\
\hline 12 & data \\
\hline 8 & Process \\
\hline 7 & design \\
\hline 7 & Management \\
\hline 5 & Lifecycle \\
\hline 4 & Engineering \\
\hline 4 & information \\
\hline 4 & maintenance \\
\hline 3 & business \\
\hline 3 & model \\
\hline 3 & PLM \\
\hline 3 & Projects \\
\hline 3 & provide \\
\hline 3 & requirements \\
\hline 3 & support \\
\hline 3 & tools \\
\hline
\end{tabular}

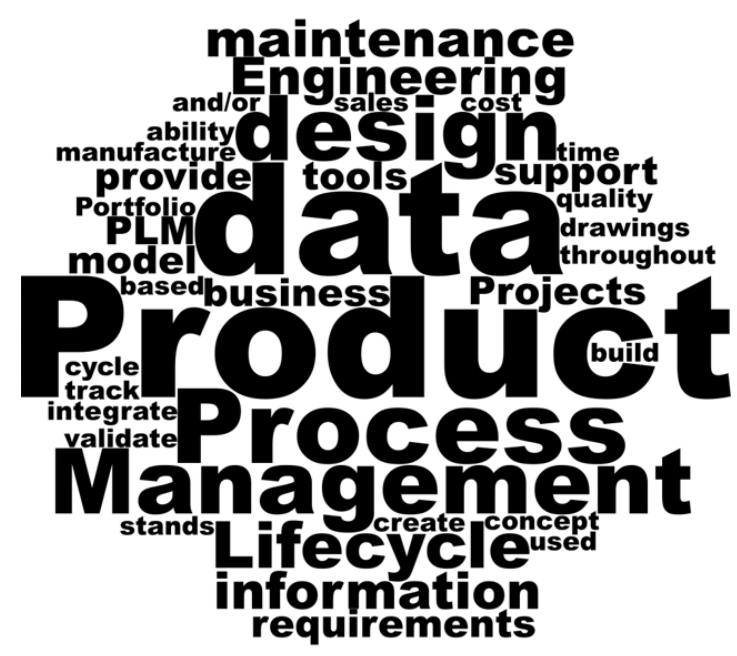

Figure 2. A consolidated word cloud analyzing word frequency for expert users when asked to define PLM

\section{Results}

Through using the frequency of words from users, a user centric definition of PLM might exist as:
Product Lifecycle Management is defined as the strategic management of data through business processes to leverage the information across the product lifecycle from design engineering, through maintenance of the product.

Through using the frequency of words from consultants, a consultant centric definition of PLM could be:

Product Lifecycle Management is defined as the systems, software, and tools used across the business enterprise to manage the processes and information required to support the creation of product data through ideation, manufacturing, and support.

From these two definitions, one can see that the users of PLM systems are clearly more focused on the design aspect of PLM. The word design was used a total of seven times by the users defining PLM, where the consultants only mentioned the word design once. The users seem to be more focused on the data from the perspective of engineering, which is not surprising considering that most PLM systems are typically deeply engrained within the engineering departments of companies.

The consultants on the other hand seem to have a broader view of PLM. For example, the word "ideation" was mentioned 3 times by the consultants and not at all by the users. This implies that the consultants view of PLM spreads further in the new product development process than the users care to define it.

From comparing these definitions to the holistic PLM definition proposed by Corallo et al., [21], we see evidence of all of the themes of PLM, although some concepts are implied and not explicitly stated. This finding corroborates the holistic definition of PLM proposed by Corallo et al., [21]. This serves as validation of this work by both actual users and consultants of PLM systems. However, we also see from the research presented in this study that the focus of PLM shifts depending upon the perspective of the person who is defining PLM. An effective implementation of PLM within a company is so broad that it may not be possible to have one unified definition, rather it may have a different definitions depending upon the perspective of the person, department, and role of the individual defining PLM. A company or implementing consultant may find that creating one central generic mission statement for the company with respect to PLM and supporting it with departmental definitions may be more relevant and successful than trying to develop one comprehensive PLM definition.

\section{Discussion}

The user centric definition of PLM is perhaps more focused on the execution of the new product development process, while the consultant view of PLM is more consistent with an enterprise view of the PLM solution. This is supported by two facts: 1 . Consultants mentioned the word enterprise 4 times in their commentary, and the users did not mention the word enterprise at all and 2. The users used the word design seven times, while the consultants used design only once. This clearly illustrates that part of the confusion with defining PLM comes from the different perspectives of the individuals involved in 
the conversation. Consultants tend to view PLM as an enterprise solution, beginning with ideation and ending with the support of the product. Users tend to focus on the PLM system to actively manage design data. Perhaps the consultant definition of PLM is broader, but the user definition of PLM is deeper?

It is intuitive that the consultants would have a larger view of PLM, as they are the visionaries and implementers of the PLM systems of the future. It also makes sense that the users would focus on the design data, as this is the core of the data that is being managed by the systems. Consultants in the PLM realm should use this research to understand that while bringing new features and functionality to PLM is fine, what is important to the users is the product design data. PLM users should realize that while gaining control of design data is very important and can yield many opportunities to save both time and money, there are other opportunities to improve the enterprise by leveraging PLM across the enterprise.

More work should be employed to create definitions of PLM across different industries as well as different organizational maturity levels. For example, a company without any PDM may be more fixated on controlling their data, while more mature companies may be more focused on integration with suppliers and other areas of opportunity specific to that company.

Companies implementing a PLM solution must recognize that PLM is not a "silver bullet" solution to fix all problems. Studies have shown that even though implementing new technological solutions may make sense from the perspective that users should be able to save time and make better decisions, companies still must investigate and optimize how the data is actually used within the company [5,22]. For example, because PLM systems are typically used to communicate data and intent between users, it is possible to create too much automation. Information overload within PLM systems is a possibility, which must be balanced against the intent of the implementation [23].

Companies may be tempted to jump into a PLM implementation because of the benefits realized by other companies. However, it is important to realize that the benefits received by PLM are also cultural in nature. The workforce must be willing to accept new changes to the way they receive and process work for any PLM implementation to be successful [24].

\section{References}

[1] C. W. Elverum and T. Welo, "On the use of directional and incremental prototyping in the development of high novelty products: Two case studies in the automotive industry," J. Eng. Technol. Manag. - JET-M, vol. 38, pp. 71-88, 2015.

[2] Y. hsiang I. Chang and C. L. Miller, "PLM curriculum development: Using an industry-sponsored project to teach manufacturing simulation in a multidisciplinary environment," J. Manuf. Syst., vol. 24, no. 3, pp. 171-177, 2006.

[3] NA, "CIMdata Publishes PLM Market and Solution Provider Report - CIMdata," cimdata.com, 2017. [Online]. Available: https://www.cimdata.com/en/news/item/8403-cimdata-publishesplm-market-and-solution-provider-report. [Accessed: 10-Apr2018].
[4] A. C. Guide, T. O. Making, S. Investments, I. N. Product, and L. Management, "Part 1: Defining PLM - Executive overview," pp. 1-5.

[5] E. Bendoly, E. D. Rosenzweig, and J. K. Stratman, "The efficient use of enterprise information for strategic advantage: A data envelopment analysis," J. Oper. Manag., vol. 27, no. 4, pp. 310-323, 2009.

[6] A. Bouras, B. Eynard, S. Foufou, and K. D. Thoben, "Product lifecycle management in the era of internet of things: 12th IFIP WG 5.1 international conference, PLM 2015 doha, Qatar, October 19-21, 2015 revised selected papers," IFIP Adv. Inf. Commun. Technol., vol. 467, no. October, 2015.

[7] F. Segonds, F. Mantelet, N. Maranzana, and S. Gaillard, "Early stages of apparel design: How to define collaborative needs for PLM and fashion?,’ Int. J. Fash. Des. Technol. Educ., vol. 7, no. 2 , pp. 105-114, 2014.

[8] M. Bordegoni, Product Virtualization: An Effective Method for the Evaluation of Concept Design of New Products. 2011.

[9] M. W. Grieves, "Product Lifecycle Management. Towards Knowledge-Rich Enterprises," vol. 388, no. July 2012, 2012.

[10] M. Huhtala, M. Lohtander, and J. Varis, "Product Lifecycle Management for a Global Market," vol. 442, no. July, 2014.

[11] L. Rivest, A. Bouras, and L. Borgen, Product Lifecycle Management: Towards Knowledge-Rich Enterprises IFIPWG 5.1 International Conference, PLM 2012. 2012.

[12] B. K. Wong, "PLM â€TM s Growing Pains," no. January, pp. 40-43, 2006

[13] M. Cantamessa, F. Montagna, and P. Neirotti, "Understanding the organizational impact of PLM systems: evidence from an aerospace company," Int. J. Oper. Prod. Manag., vol. 32, no. 2, pp. 191-215, 2012.

[14] J. Brown, "Strategies of the Major PLM Vendors 2016+ - TechClarity," 2016. [Online]. Available: https://tech-clarity.com/strategies-of-the-major-plm-vendors2016/5048. [Accessed: 10-Apr-2018].

[15] O. Shilovitsky, "Beyond PLM (Product Lifecycle Management) Blog PLM Definition from Dassault System's Al Bunshaft Beyond PLM (Product Lifecycle Management) Blog," beyondplm.com, 2011. [Online]. Available: http://beyondplm.com/2011/08/15/plm-definition-from-dassaultsystems-al-bunshaft/. [Accessed: 10-Apr-2018].

[16] V. Gecevska, T. Stojanova, and B. Jovanovski, "Product lifecycle management tools," Ann. Fac. Eng. Hunedoara - Int. J. Eng., vol. 1, pp. 219-222, 2013.

[17] "PLM Software / Product Lifecycle Management Software." [Online]. Available:

https://www.plm.automation.siemens.com/global/en/ourstory/glossary/product-lifecycle-management-plm-software/12506. [Accessed: 10-Apr-2018].

[18] "Windchill | PLM | PTC." [Online]. Available: https://www.ptc.com/en/products/plm/plm-products/windchill. [Accessed: 10-Apr-2018].

[19] "What is PLM? | Product Lifecycle Management | Autodesk." [Online]. Available: https://www.autodesk.com/content/productlifecycle-management. [Accessed: 10-Apr-2018].

[20] J. Stark, "John Stark Associates | PLM services and publications." [Online]. Available: http://www.johnstark.com/. [Accessed: 10Apr-2018].

[21] A. Corallo, M. E. Latino, M. Lazoi, S. Lettera, M. Marra, and S. Verardi, "Defining Product Lifecycle Management: A Journey across Features, Definitions, and Concepts," ISRN Ind. Eng., vol. 2013, pp. 1-10, 2013.

[22] T. Davenport, "Back to Decision-making basics," 2008. [Online]. Available: https://hbr.org/2008/03/back-to-decisionmaking-basics. [Accessed: 04-Oct-2018].

[23] A. G. Gunendran and R. I. M. Young, "Methods for the capture of manufacture best practice in product lifecycle management," Int. J. Prod. Res., vol. 48, no. 20, pp. 5885-5904, 2010.

[24] H. Kärkkäinen, H. J. Pels, and A. Silventoinen, "Defining the customer dimension of PLM maturity," IFIP Adv. Inf. Commun. Technol., vol. 388 AICT, no. July, pp. 623-634, 2012. 